\title{
Comparison of Olsen and Barrett-universal II; two intraocular lens power calculation formulas for monofocal intraocular lens, in Iranian patients; Kermanshah; 2018-2020
}

\section{ashkan safarzadehkhoushabi ( $\square$ ashkans716@gmail.com )}

Department of Ophthalmology, Imam Khomeini hospital, Kermanshah University of medical sciences and health services, Kermanshah, Iran

mojtaba eidizadeh

Department of Ophthalmology, Imam Khomeini hospital, Kermanshah University of medical sciences and health services, Kermanshah, Iran

leila ebadi-soofloo

Department of Ophthalmology, Imam Khomeini hospital, Kermanshah University of medical sciences and health services, Kermanshah, Iran

\section{Research Article}

Keywords:

Posted Date: February 24th, 2022

DOI: https://doi.org/10.21203/rs.3.rs-1342174/v1

License: (c) (i) This work is licensed under a Creative Commons Attribution 4.0 International License. Read Full License 


\section{Abstract}

Background: With improvements in the formulas, the accuracy of predicting IOL power in emmetropic eyes with an axial length (AL) of 22.0 to $24.5 \mathrm{~mm}$ can now be guaranteed. On the other hand, as the condition of the fundus is complex in highly myopic eyes, many formulas are unable to predict the IOL power accurately; in such cases. Myopia, associated with long axial length (AL), is a global public health issue. The estimated incidence of myopia has been reported between 25 to $46.4 \%$. The IOL power calculation formulas are less accurate in long eyes, which is commonly defined as AL longer than 24.5 $\mathrm{mm}$.

Material and methods:This prospective cohort study protocol was reviewed and approved by the local university Institutional Ethics Committee and the tenets of the Declaration of Helsinki were followed throughout the study. A written informed consent was obtained from each patient and only those who were consenting and willing for follow-up were enrolled into the study.

Results: Before being included in the study, all patients were informed of its purpose and gave their written consent. The study protocol was approved by the Ethics Committee of the Kermanshah University Medical Sciences and health services, and the study complied with the tenets of the Declaration of Helsinki. Institutional review board approval was obtained for this study. Of the 423 eyes underwent cataract surgery during the study period, Inclusion criteria were met by 250 eyes. 173 eyes were excluded from the study due to inadequate biometry data $(\mathrm{N}=136)$; associated ocular comorbidities and previous intraocular surgeries $(\mathrm{N}=19)$ or postoperative complications $(\mathrm{N}=18)$.

Discussion:In our cohort study on 250 eyes in Iranian patients during 2018 to 2020 , the BU II formula was found to be most accurate than Olsen in normal and short axial length eyes; neither long axial length eyes. It also had the lowest MAE, standard deviation of error, median absolute error, and highest percentage of eyes within $\pm 0.25 \mathrm{D}, \pm 0.50 \mathrm{D}$, and $\pm 1.00 \mathrm{D}$; all significantly meaningful ( $p$-value $<0.005$ ).

Conclusion:Our study on 250 Iranian eyes underwent phacoemulsification surgery (microincision cataract surgery) with posterior chamber IOL implantation, and received an in-the-bag insertion of a monofocal IOL between March 2018 to August 2020. After measurement of some refractive information with Lenster L900; data analyses revealed that BUII formula perfoming more accurately than Olsen formula in eyes with normal or short axial length.

\section{Introduction}

Cataract surgery known as one of the most common procedures. Lots of developments have led to improved outcomes after intraocular lens (IOL) implantation. Development of new IOL types (1); also investigation of new IOL power calculation formulas(2); are the leading causes to achieve the best postoperative refraction. 
Nowadays; there is no global consensus about which formula calculates the most accurate refractive prediction in all patients with any type of ocular structure. Some authors have mentioned that surgeons should use different formulas for eyes of varied ocular dimensions $(3,4)$.

Performing best formula; helps to achieve the patient's expectation to be free of spectacle use. This is especially useful for patients who previously underwent refractive surgeries.

With improvements in the formulas, the accuracy of predicting IOL power in emmetropic eyes with an axial length (AL) of 22.0 to $24.5 \mathrm{~mm}$ can now be guaranteed. On the other hand, as the condition of the fundus is complex in highly myopic eyes, many formulas are unable to predict the IOL power accurately; in such cases(5). Myopia, associated with long axial length (AL), is a global public health issue. The estimated incidence of myopia has been reported between $25(6)$ to $46.4 \%(7)$. The IOL power calculation formulas are less accurate in long eyes, which is commonly defined as AL longer than $24.5 \mathrm{~mm}(8-11)$. in the United States and even higher in some parts of Asia(12). however, recent studies revealed that formulas including Barrett Universal II, Olsen, Haigis, were more accurate, not only in emmetropic eyes, but also in long axis eyes $(10,13)$.

Methods for accurately calculating IOL power in normal and complex eyes are evolving. The accuracy of new-generation formulas used to calculate IOL power, such as the Barrett Universal II and Olsen, requiring the measurement of more parameters, has drawn the attention of surgeons.

Olsen formula, uses exact ray tracing technique and thick-lens considerations for IOL power calculation, also include a $\mathrm{C}$ constant that indicates the final position of $\mathrm{IOL}(14)$.

The Barrett Universal II (BU-II) formula, an updated version of BU formula, was introduced since 2010 by Graham D Barrett and has shown promising results so far $(15,16)$. This formula can be accessed in the online form in Asia Pacific Association of Cataract and Refractive Surgeons website (16). Recent studies reporting large series $(10,13)$ have revealed the Barrett Universal II formula to have the minimal absolute error compared with other modern formulas. The Barrett Universal(17) (Barrett) and the Olsen(18) formulas are more accurate than older generation formulas $(10,19)$.

Both formulas similarly, being based on thin-lens optical principles, and all require the axial length (AL) and corneal power and preoperative anterior chamber depth (ACD) to predict the estimated lens position (ELP), which is the estimated postoperative distance between the anterior corneal surface and the principal plane of a thin IOL.

In 2011 , a large series reported $71 \%$ and $95 \%$ of cases were within 0.5 and $1.0 \mathrm{D}$ of predicted refraction(2). in comparison, a more recent large multicenter study reported up to $81 \%$ and $98 \%$ of eye; respectively(13). In both studies; patients underwent IOL implantation, performing Olsen and BU II formulas to calculate the IOL power.

The aim of current study was to assess the accuracy of these new IOL formulas in Iranian population; to revealing which is the best overall predictor of the actual postoperative refractive outcome. 
A subgroup analysis will also examine each AL subgroup and differing IOL types.(Table 1)

Table 1

Summary of intraocular lens formulas

\begin{tabular}{|llll|}
\hline Formula & First Publication & Metrics Used & Derivation Method \\
\hline SRK/T & 1990 & AL, K & Theoretical \\
\hline Hoffer Q & 1993 & AL, K & Theoretical \\
\hline Haigis & 1993 & AL, K, ACD & Theoretical \\
\hline Barrett 1 & 1993 & AL, K, ACD & Theoretical \\
\hline Holliday 1 & 1998 & AL, K & Theoretical \\
\hline Olsen & 2007 & AL, K, ACD, LT, CCT & Ray tracing \\
\hline Barrett 2 & 2016 & AL, K, ACD, LT, WTW & Theoretical \\
\hline Hill-RBF 1.0 & 2016 & AL, K, ACD & Regression/artificial intelligence \\
\hline Hill-RBF 2.0 & 2018 & AL, K, ACD, WTW, LT, CCT & Regression/artificial intelligence \\
\hline Holliday 2 & 2018 & AL,K,ACD, LT,WTW,age,PR & Theoretical \\
\hline Kane & 2018 & AL, K, ACD, sex, LT, CCT & Theoretical/artificial intelligence \\
\hline
\end{tabular}

$\mathrm{ACD}=$ anterior chamber depth; $\mathrm{AL}=$ axial length; $\mathrm{CCT}=$ central corneal thickness; $\mathrm{K}=$ keratometry; $\mathrm{LT}=$ lens thickness; $\mathrm{PR}=$ preoperative refraction; $\mathrm{WTW}=$ white to white

\section{Material And Methods}

This prospective cohort study protocol was reviewed and approved by the local university Institutional Ethics Committee and the tenets of the Declaration of Helsinki were followed throughout the study. A written informed consent was obtained from each patient and only those who were consenting and willing for follow-up were enrolled into the study.

The medical records of all patients suffering visually significant cataract in the age group between 40 to 80 years, were evaluated for this study. Patients included in this article; were underwent an uncomplicated cataract surgery with posterior chamber IOL implantation, and received an in-the-bag insertion of a monofocal IOL between March 2018 to August 2020.

All surgeries were done by one experienced cataract surgeon in the Department of Ophthalmology, ImamKhomeini hospital, Kermanshah University of medical sciences and health services, Kermanshah, Iran.

Eyes with these conditions were included in the study: no additional ocular surgery, no known ocular pathology other than cataract (significant corneal scarring, keratoconus or other ectasia, keratoplasty, past laser vision correction, corneal relaxing incisions), complete preoperative data, no intraoperative 
complication (anterior or posterior capsule tear, vitreous prolapse or zonular dehiscence, postoperative corrected distance visual acuity lower than $0.8(20 / 25)$ ), availability of corrected distance visual acuity (CDVA), and subjective refraction pre-operatively as well as 3 months after phacoemulsification and this type of IOL (20)). No eyes were excluded because of unexpected refractive outcomes.

Preoperative and postoperative keratometry $(K)$ and axial length $(A L)$, anterior chamber depth $(A C D)$, and horizontal white-to-white (WTW) distance were all measured using Lenstar 900 (Haag-Streit AG, Koeniz, Switzerland). Other data were collected from medical records. All patients examined by 3 experienced cataract surgeons before underwent surgery.

All patients underwent uncomplicated cataract surgery with an implantation of nontoric nonmultifocal IOL at our institution (SN60WF) through a clear cornea temporal incision phacoemulsification (21).

If both eyes were suitable conditions and vision was unequal after surgery, the eye was chosen with greater visual clarity. If both eyes were eligible and visual acuity was equal, the first eye was selected. A random eye was chosen if immediate sequential bilateral surgery was performed.

Postoperative assessment included subjective manifest refraction ( $6 \mathrm{~m}$ refractive lane; Snellen chart) and was obtained 3 months after surgery.

Calculating the IOL power performed by The Barrett Universal II online calculator (version 1.05) (21) from the Asia-Pacific Association of Cataract \& Refractive Surgeons; or predicted refraction for the Olsen formula with the Phaco-optics program (version 1.10.100.2032); respectively(22).

The Barrett Universal II formula only needs AL and corneal power measurements. For the Barrett Universal II, it is nonessential but recommended that the ACD, WTW distance, and the lens thickness are additionally in-putted because they might contribute to more accurate results. The ACD is defined as the distance from the corneal epithelium to the lens(23). Parameters were used for the Olsen formula included: $K$ values, AL, ACD, preoperative refraction, WTW distance, and central corneal thickness.

The lens constant resulting in a mean refractive prediction error as close to zero as possible was calculated for every formula.

For each patient and formula, an optimized lens constant was determined, which resulted in the individual difference between the predicted spherical equivalent (SE) and the actual SE to be zero. The optimized lens constants for the Barrett Universal II (lens factor) and Olsen (ACD-constant) were determined by trial and error, varying it in 0.01 steps.

Lens constant deviated by more than 2 standard deviations (SDs) from the population mean, was not included for calculating the overall optimized lens constant. The optimized lens constant was calculated as the mean of all remaining individual patients' constants. 
Mean absolute errors (MAE) was selected as the primary outcome measure since the positive and negative prediction error could cancel each other out $(24,25)$. MAE was the average of the absolute prediction error value, which was back-calculated by subtracting the post-operative actual refractive sphere equivalent from the calculated refractive sphere equivalent generated by each formula(24). However, the percentage of eyes within $0.50 \mathrm{D}$ of prediction error was secondary outcome of this study. As another indicator of $\mathrm{IOL}$ power calculation accuracy.

The refractive prediction error is defined as the measured postoperative SE refraction minus the predicted SE calculated by the formula. A positive prediction error stands for a refractive outcome that is more hyperopic than predicted, whereas a negative prediction error indicates a more myopic outcome. The mean absolute error (MAE), SD, median absolute error, maximum absolute error, as well as percentages of eyes within prediction error targets of $0.25 \mathrm{D}, 0.50 \mathrm{D}, 1.00 \mathrm{D}$, and 2.00 D were determined for each formula. The formulas were ranked by the MAE.

Eyes were separated into subgroups based on axial length: short $(\leq 22.0 \mathrm{~mm})$, medium $(>22.0$ to $<24.0$ $\mathrm{mm}$ ) and long ( $\geq 24.0 \mathrm{~mm})$.

Statistical analysis was performed with Stata software (version 13, Statacorp LLC). Similar to Reitblat et al.(26) a sample-size calculation was conducted to detect a prediction error more than $0.125 \mathrm{D}$ and a SD of $0.30 \mathrm{D}$. A total of 52 eyes were required for a significance level of 0.05 and a test power of 0.80 . First, the Shapiro-Wilk test was performed. The Friedman test was used to determine differences in absolute errors between the formulas. In cases with a significant result, post hoc analysis was performed using the paired $t$ test or Wilcoxon signed-rank test dependent on data distribution. The Bonferroni correction was applied for multiple comparisons. Unadjusted $\mathrm{P}$ values are reported. Adjusted $\mathrm{P}$ values less than 0.05 were considered statistically significant.

\section{Results}

Before entering the study, all patients were informed of its purpose and expressed their written consent. The study protocol was approved by the Ethics Committee of the Kermanshah University Medical Sciences and health services, and the study complied with the tenets of the Declaration of Helsinki. Institutional review board approval was obtained for this study. Of the 423 eyes underwent cataract surgery during the study period, Inclusion criteria were met by 250 eyes. 173 eyes were excluded from the study due to inadequate biometry data $(\mathrm{N}=136)$; associated ocular comorbidities and previous intraocular surgeries $(\mathrm{N}=19)$ or postoperative complications $(\mathrm{N}=18)$.

As all eyes were operated by the same surgeon, constant optimization did not have to compensate for different surgical techniques, such as different capsulorhexis size, influencing the postoperative IOL position. On the other hand the postoperative refraction was assessed by the same surgeon, who measured it with the maximum attention. 
Patients separated in 3 study groups according to ALs: 135 eyes in normal AL group [AL 22-24.5 mm], 53 eyes in short $A L$ groups [ $A L<22 \mathrm{~mm}$ ], and 62 eyes in long $A L$ groups [AL $>24.5 \mathrm{~mm}$ ].

The mean age of the study group population was $61.8 \pm 2.73$ years (range: $59-64$ years), with 141 male patients (mean age 61.9 \pm 3.45 years; range 61-64 years) and 109 female patients (mean age 60.2 \pm 2.82 years; range 59-62 years). The mean age of the study patients in normal AL group was $61.29 \pm 2.51$ years, short AL group was $62.24 \pm 3.78$ years, and those in long AL group was $61.19 \pm 3.28$ years.

The mean preoperative visual acuity was $20 / 50 \pm 20 / 50$ LogMAR units in the whole study population, with $10 / 50 \pm 20 / 50,30 / 50 \pm 10 / 50$, and 20/50 $\pm 30 / 50$ in normal AL group, short AL group, and long AL group, respectively. (P-value $>0.05)$.

The mean uncorrected visual acuity (UCVA) and mean best corrected visual acuity (BCVA) of the study subjects at 3 month postoperative follow up are summarized in Table 5.

In the entire study sample population, the BU II formula gave the lowest mean absolute error and median absolute error in predicted postoperative refraction. This was noted in the individual groups as well, with mean absolute error of $0.32 \pm 0.25 \mathrm{D}$ in normal AL group, $0.45 \pm 0.24 \mathrm{D}$ in eyes with $A L<22 \mathrm{~mm}$, and 0.38 $\pm 0.23 \mathrm{D}$ in eyes with $\mathrm{AL}>24.5 \mathrm{~mm}$.

Statistically significant differences were observed between the mean absolute errors in postoperative refraction given by the two formulas (by Freidman test) in normal and short axial length groups. BU II gave the lowest mean absolute prediction error in postoperative refraction and median absolute error in normal and short AL group, respectively.

The mean postoperative refractive error (spherical equivalent) at 3 month was $0.20 \pm 0.49 \mathrm{D}$ (range: $0.63 \mathrm{D}$ to $1.13 \mathrm{D})$.

The comparison (by post hoc analysis using Wilcoxon signed ranks test) of the mean absolute prediction errors between the IOL formulas in the 3 study groups is summarized in Table 4. In all the three AL groups, there was statistically significant difference between mean prediction error in postoperative refraction of Barrett and BU II (P value < 0.0005); however, the difference in mean absolute prediction errors between Olsen and BU II formulas was statistically significant in the short AL group ( $P$ value < $0.0005)$, also in the normal $A L$ group ( $P$ value $<0.0005)$. In the long axial length eyes group, there was no statistically significant difference between formula $(p=0.24)$. No post-hoc analysis was performed because of the lack of statistically significant result.

Eyes with prediction error in postoperative refraction within $\pm 1 \mathrm{D}$ and $\pm 0.5 \mathrm{D}$ of the given target by the two formulas was highest with BU II formula with approximately $98 \%$ and $71 \%$ of the eyes achieving within $\pm 1 \mathrm{D}$ and $\pm 0.5 \mathrm{D}$ of the given target postoperative refraction, respectively.

The mean implanted IOL power was $22.29 \mathrm{D} \pm 4.23(\mathrm{SD})$ (range 10.5 to $31.5 \mathrm{D}$ ). 
Our study revealed the Barrett Universal II had the lowest MAE. There was a statistically significant difference in absolute error between the two IOL power calculation formulas $(P<.001)$. Compared with the Barrett Universal II formula Olsen ranked worse. The BU II formula provided predictions that were more accurate than those using the Olsen in normal and short AL grupe $(P<.001)$.

Significantly more eyes placed in PE between - 25 D _+.25 D with BU II formula.

This findings emphasized on accuracy of BU II formula except in long AL patient.

Repeated-measures ANOVA did not reveal any statistically significant difference for the mean PE (P $=.2738$ ), which was close to zero with all formulas due to constant optimization.

The most interesting finding is the lack of far outliers for Barrett formula.

Table 6 shows the change in biometric variables between preoperatively and postoperatively. The $\mathrm{K}$ flat, $\mathrm{K}$ steep, and AL values did not change significantly after the IOL implantation; however, there was a significant decrease in the ACD (P Z .008).

The simple linear regression used to predict the pre-operative ACD from the postoperative value found statistically significant regression $(P=.002)$. The correlation coefficient $(R 2)$ was 0.373 based on the following equation:

ACD preoperative $=1.95 * 0.505 * A C D$ postoperative.

The difference between the preoperative and postoperative values was not statistically significant. Thus, if the mean preoperative values were considered as references for IOL power calculation and because the changes in the values (flat K: D; steep K: D; AL: mm) was not significant.

Table 5 showed the refractive errors and visual acuity before and after surgery.

Table 2 shows the characteristics of the study population.

Table 2

Demographic data of the patients by axial length group.

\begin{tabular}{|lllll|}
\hline Parameter & short AL & normal AL & long AL & P Value \\
& $<22 \mathrm{~mm}$ & $\mathbf{2 2 . 0 - 2 4 . 5 ~ m m}$ & $>\mathbf{2 4 . 5} \mathbf{m m}$ & \\
\hline Mean age (year) & $62.24 \pm 3.78$ & $61.29 \pm 2.51$ & $61.19 \pm 3.28$ & .07 \\
\hline Sex (Male / Female) & $46 / 23$ & $54 / 49$ & $41 / 37$ & .47 \\
\hline Eye (Right / Left) & $33 / 44$ & $45 / 45$ & $41 / 42$ & .52 \\
\hline No significant differences were found between demographic data in 3 groups. \\
\hline
\end{tabular}


Table 3 revealed Refractive measurements obtained with the formulas investigated between three study groups. Differences between two formulas was significantly lower in BU II group versus Olsen group in normal and short axial length groups.

This meaningful accuracy was not obtained in long axial length group.

Table 3

Refractive outcomes obtained with the formulas investigated and the biometric measurements.

\begin{tabular}{|c|c|c|c|c|}
\hline \multirow[t]{2}{*}{ Formula } & Mean Absolute & Median Absolute & Range (D) & \multirow[t]{2}{*}{$P$ value } \\
\hline & Error \pm SD (D) & \multicolumn{2}{|l|}{ Error (D) } & \\
\hline \multicolumn{5}{|c|}{ Entire Study Population } \\
\hline Olsen & $0.38 \pm 0.27$ & 0.39 & 1.31 to 1.63 & \multirow[t]{2}{*}{$<0.0005$} \\
\hline BU II & $0.27 \pm 0.38$ & 0.29 & 1.29 to 1.38 & \\
\hline \multicolumn{5}{|c|}{ Eyes with Normal Axial Length } \\
\hline Olsen & $0.35 \pm 0.28$ & 0.35 & 1.3 to 1.6 & \multirow[t]{2}{*}{$<0.0005$} \\
\hline BU II & $0.37 \pm 0.25$ & 0.30 & 1.31 to 1.43 & \\
\hline \multicolumn{5}{|c|}{ Eyes with Short Axial Lengths } \\
\hline Olsen & $0.54 \pm 0.38$ & 0.59 & 0.80 to 1.6 & \multirow[t]{2}{*}{$<0.0005$} \\
\hline BU II & $0.35 \pm 0.24$ & 0.28 & 0.46 to 1.2 & \\
\hline \multicolumn{5}{|c|}{ Eyes with Long Axial Lengths } \\
\hline Olsen & $0.39 \pm 0.39$ & 0.35 & 0.81 to 0.21 & \multirow[t]{2}{*}{0.240} \\
\hline BU II & $0.37 \pm 0.23$ & 0.26 & 0.54 to 0.75 & \\
\hline
\end{tabular}


Table 4

demonstrated Optimized Constant and Lens constant for both formulas.

\begin{tabular}{|c|c|c|c|c|c|c|c|}
\hline formulas & $\begin{array}{l}\text { Optimized } \\
\text { Constant }\end{array}$ & $\begin{array}{l}\text { Lens } \\
\text { constant }\end{array}$ & $\mathrm{PE} \pm \mathrm{SD}$ & $\begin{array}{l}\mathrm{PE} \leq \\
0.25 \mathrm{D}\end{array}$ & $\begin{array}{l}P E \leq \\
0.50 \mathrm{D}\end{array}$ & $\begin{array}{l}\mathrm{PE} \leq \\
0.75 \mathrm{D}\end{array}$ & $\begin{array}{l}\mathrm{PE} \leq \\
1.00 \mathrm{D}\end{array}$ \\
\hline \multicolumn{8}{|c|}{ Entire study group } \\
\hline Barrett & 118.5 & $\begin{array}{l}\text { Lens } \\
\text { factor }\end{array}$ & $\begin{array}{l}0.005 \pm \\
0.323\end{array}$ & 62.05 & 93.20 & 95.33 & 100.0 \\
\hline Olsen & 4.45 & ACD & $\begin{array}{l}0.010 \pm \\
0.326\end{array}$ & 60.58 & 90.33 & 90.00 & 98.04 \\
\hline \multicolumn{8}{|c|}{ short AL group } \\
\hline Barrett & 118.5 & $\begin{array}{l}\text { Lens } \\
\text { factor }\end{array}$ & $\begin{array}{l}0.015 \pm \\
0.302\end{array}$ & 64.45 & 90.20 & 94.43 & 100.0 \\
\hline Olsen & 4.45 & ACD & $\begin{array}{l}0.014 \pm \\
0.325\end{array}$ & 58.58 & 77.33 & 81.88 & 97.11 \\
\hline \multicolumn{8}{|c|}{ Normal AL group } \\
\hline Barrett & 118.5 & $\begin{array}{l}\text { Lens } \\
\text { factor }\end{array}$ & $\begin{array}{l}0.005 \pm \\
0.311\end{array}$ & 66.15 & 87.21 & 94.31 & 99.89 \\
\hline Olsen & 4.45 & ACD & $\begin{array}{l}0.012 \pm \\
0.325\end{array}$ & 59.48 & 84.27 & 88.72 & 95.14 \\
\hline \multicolumn{8}{|c|}{ Long AL group } \\
\hline Barrett & 118.5 & $\begin{array}{l}\text { Lens } \\
\text { factor }\end{array}$ & $\begin{array}{l}0.005 \pm \\
0.329\end{array}$ & 61.15 & 88.19 & 94.23 & 98.26 \\
\hline Olsen & 4.45 & ACD & $\begin{array}{l}0.015 \pm \\
0.317\end{array}$ & 60.56 & 87.29 & 93.20 & 98.14 \\
\hline
\end{tabular}

$\mathrm{AL}=$ axial length; $\mathrm{EVO}=$ Emmetropia Verifying Optical; $\mathrm{MAE}=$ mean absolute error; $\mathrm{MedAE}=$ median absolute error; $\mathrm{PE}=$ prediction error; RBF = Radial Basis Function.

*Significantly different compared with the Barrett Universal II formula $(P<0.05$, related-samples Friedman 2-way analysis of variance by rank) 
Table 5

Preoperative and postoperative patient characteristics.

\begin{tabular}{|llll|}
\hline Parameter & Mean \pm SD & Minimum & Maximum \\
\hline Preoperative & & & \\
\hline Sphere (D) & $9.89 \pm 4.03$ & 5.32 & 13.85 \\
\hline Cylinder (D) & $2.43 \pm 1.59$ & 0.00 & 4.23 \\
\hline SE (D) & $8.69 \pm 3.58$ & 3.13 & 12.02 \\
\hline BCVA & $40 / 50$ & $30 / 50$ & $20 / 20$ \\
\hline CDVA & $30 / 50$ & $20 / 50$ & $20 / 20$ \\
\hline UCDVA & $25 / 50$ & $10 / 50$ & $20 / 20$ \\
\hline Postoperative & & & \\
\hline Sphere (D) & $0.46 \pm 0.62$ & 1.69 & 0.49 \\
\hline Cylinder (D) & $0.59 \pm 0.54$ & 0.00 & 2.02 \\
\hline SE (D) & $0.20 \pm 0.49$ & 1.13 & 0.63 \\
\hline BCVA & $25 / 30$ & $25 / 30$ & $20 / 20$ \\
\hline UDCVA & $25 / 30$ & $20 / 30$ & $20 / 20$ \\
\hline CDVA & $25 / 30$ & $20 / 30$ & $20 / 20$ \\
\hline CDVA = corrected distance visual acuity; SE = spherical equivalent \\
\hline
\end{tabular}

Table 6

shows the change in biometric variables between preoperatively and postoperatively. The $\mathrm{K}$ flat, $\mathrm{K}$ steep, and $\mathrm{AL}$

\begin{tabular}{|llllll|}
\hline Parameter & Preoperative & \multicolumn{3}{c}{ Postoperative } & \multirow{2}{*}{ P Value } \\
\cline { 2 - 5 } & Mean \pm SD & Range & Mean \pm SD & Range & \\
\hline K flat (D) & $41.48 \pm 1.47$ & $36.79-44.77$ & $41.28 \pm 1.56$ & $37.12-44.88$ & .052 \\
\hline K steep (D) & $42.85 \pm 1.72$ & $41.72-47.60$ & $42.92 \pm 1.67$ & $41.74-48.76$ & .53 \\
\hline AL $(\mathrm{mm})$ & $23.22 \pm 1.71$ & $23.12-30.41$ & $27.35 \pm 2.6$ & $22.63-31.53$ & .90 \\
\hline ACD $(\mathrm{mm})$ & $3.28 \pm 0.32$ & $2.87-4.52$ & $3.31 \pm 0.56$ & $2.27-4.45$ & $.008^{\star}$ \\
\hline
\end{tabular}

$\mathrm{ACD}=$ anterior chamber depth; $\mathrm{AL}=$ axial length; $\mathrm{K}=$ keratometry

*Statistically significant difference $(\mathrm{P}<.005)$ 
The ACD was the only biometric parameter that changed significantly after pIOL implantation in our study

\section{Discussion}

In our cohort study on 250 eyes in Iranian patients during 2018 to 2020, the BU II formula was found to be most accurate than Olsen in normal and short axial length eyes; neither long axial length eyes. It also had the lowest MAE, standard deviation of error, median absolute error, and highest percentage of eyes within $\pm 0.25 \mathrm{D}, \pm 0.50 \mathrm{D}$, and $\pm 1.00 \mathrm{D}$; all significantly meaningful ( $p$-value $<0.005$ ).

Multiple studies $(13,19,27)$ have shown the Barrett Universal 2 formula is more accurate than the thirdgeneration and Olsen formulas, which was confirmed in this study. Barrett formula confirmed its reputation as being one of the most accurate, as previously found by many researchers $(10,13,19)$.

The present investigation was designed to assess the refractive outcomes of IOL power calculation using the measurements provided by BU II and Olsen formulas. This finding demonstrates the improved accuracy of IOL power calculation.

As the Savini et al. confirmed; The most interesting finding is the lack of far outliers for BU II formula (28).

The outcomes we obtained exceeded our expectations. Because of both formulas achieved a PE of \pm 0.50 D or less, higher than $90 \%$.

Even more interestingly, with both formulas, more than $55 \%$ of eyes had a PE within 0.25 D: we should remember that according to the benchmark established by the National Health Service of the United Kingdom, this percentage should be reached for eyes with a PE within 0.50 and not $0.25 \mathrm{D}(29)$.

Moreover, our sample included eyes with an AL shorter than $22.0 \mathrm{~mm}$. short eyes are known to have poorer refractive outcomes(10), which can influence the results in the whole sample.

As a potential limitation, a smaller number of eyes were analyzed in this study than in other recent investigations, which included more than 1000 eyes $(10,13,19,30)$. Although our sample size was sufficient to detect a statistically significant difference in the MedAE (on the basis of sample size calculation), we acknowledge that big data can provide us with additional information and therefore will go on enrolling patients. A larger sample is needed to confirm our preliminary data because neither the BU II nor the Olsen formulas have been published and little is known about their structure, it is not possible to discuss the reasons for their excellent performance. However, they look promising and deserve our attention.

A recent major retrospective study (13) compared the accuracy of 7 popular IOL prediction formulas (Barrett Universal II, Haigis, Hoffer Q, Holladay 1, Holladay 2, Olsen, and SRK/T) and concluded that the Barrett, and Olsen formulas are more accurate than other formulas in eyes with AL of $24.5 \mathrm{~mm}$ or more. 
The Barrett is a 5 -variable vergence formula. Although recent studies $(13,31-33)$ reported accuracy outcomes of the Barrett formulas in long eyes.

Three previous studies (34-36) compared the Axial Length before and after insertion of pclOL and found that the difference was small and not significant. This is due to the thinness of $\mathrm{pIOL}$ and the low refractive index of acrylic the main component of pIOLs; thus, the effect on optical path length is relatively insignificant. The results in those studies are similar to our finding of a mean difference in AL of $0.029 \mathrm{~mm}$.

Reducing the Axial Length by $0.1 \mathrm{~mm}$ changes the $\mathrm{IOL}$ power by approximately $0.30 \mathrm{D}$, which is equivalent to a refractive change of $0.20 \mathrm{D}$ to $0.25 \mathrm{D}$. (37).

The higher the AL, the less accurate the measurement. To overcome this, Wang et al (38) proposed formulas for AL optimization specific for each IOL calculation formula based on results obtained at 2 centers. Furthermore, Abulafia et al (32) found that using the AL-adjusted method with the Barrett Universal II, Holladay 1, and Haigis formulas yielded the desired refractive error in eyes with an AL greater than $26.0 \mathrm{~mm}$.

The ACD was the only biometric parameter that changed significantly after pIOL implantation in our study; as Amro et al. achieved similar results (39). Shin et al. (40) compared preoperative and postoperative ocular biometry values and found that the ACD results did not correspond to the anterior iris-fixated pIOL surface were the result of the light reflection off the pIOL.

Despite present study using Lenstar L900 to achieve IOL power, some study calculating power by optical coherence tomography optical biometer. Other main limitation is the relatively small number of eyes and therefore those results cannot be widely generalized. Further studies need to be done to corroborate the results.

\section{Conclusion}

Our study on 250 Iranian eyes underwent phacoemulsification surgery (microincision cataract surgery) with posterior chamber IOL implantation, and received an in-the-bag insertion of a monofocal IOL between March 2018 to August 2020. After measurement of some refractive information with Lenster L900; data analyses revealed that BUII formula perfoming more accurately than Olsen formula in eyes with normal or short axial length.

\section{Abbreviations}

ACD: anterior chamber depth; AOD: Angle opening distance; ASOCT: Anterior segment optical coherence tomography; AT: Aspiration time; CCT: central corneal thickness; CDE: cumulative dissipated energy; IFU: Infusion fluid usage; IOP: Intraocular pressure; OHT: ocular hypertension; ONH: optic nerve head; PACG: 
Primary angle closure glaucoma; POAG: primary open angle glaucoma; PXF: Pseudoexfoliation; TISA: Trabecular-iris surface area; TM: trabecular meshwork

\section{Declarations}

\section{Acknowledgments}

We extend our thanks to clinical research development center of Imam Khomeini Hospital.

\section{Authors' contributions}

ME designed the study and performed the surgeries. ME and LE and AS conducted data collection, analysis and interpretation. ME and LE and AS wrote the manuscript. All authors read and approved the final manuscript.

\section{Funding}

This study was funded by Kermanshah University of Medical Sciences (N0. 980886).

\section{Availability of data and materials}

Consent has been obtained from all patients to enter the study (not to publish their information).

But information will be provided at your reasonable request.

The datasets generated and analyzed during the current study are not publicly available due to their containing information that could compromise the privacy of research participants but are available from the corresponding author on reasonable request.

\section{Ethics approval and consent to participate}

The Ethical Review Committee of Kermanshah University of Medical Sciences approved the study (no: IR.KUMS.REC.1399.1008). Informed written consent was taken from all participants. It was performed in accordance with the tenets of the Declaration of Helsinki.

\section{Consent for publication}

Informed written consent was taken from all participants. 


\section{Competing interests}

The authors declare that they have no competing interests.

\section{References}

1. T. K. First implantation of a diffractive quadrifocal (trifocal) intraocular lens. J Cataract Refract Surg. 2015;41:2330-2.

2. Aristodemou P KCN, Sparrow JM, et al. Intraocular lens formula constant optimization and partial coherence interferometry biometry: refractive outcomes in 8108 eyes after cataract surgery. $\mathrm{J}$ Cataract Refract Surg. 2011;37:50-62.

3. Aristodemou P KCN, Sparrow JM, Johnston RL. Formula choice: Hoffer Q, Holladay 1, or SRK/T and refractive outcomes in 8108 eyes after cataract surgery with biometry by partial coherence interferometry. J Cataract Refract Surg. 2011;37:63-71.

4. Ladas JG SA, Devgan U, Jun AS. A 3-D "super surface" combining modern intraocular lens formulas to generate a "super formula" and maximize accuracy. JAMA Ophthalmol. 2015;133:1431-6.

5. McBrien NA GA. Role of the sclera in the development and pathological complications of myopia. Prog Retin Eye Res. 2003;22:307-38.

6. Sperduto RD SD, Roberts J, Rowland M. Prevalence of myopia in the United States. Arch Ophthalmol. 1983;101:405-7.

7. McCarty CA LP, Taylor HR. Prevalence of myopia in adults: implications for refractive surgeons. $J$ Refract Surg. 1997;13:229-34.

8. Olsen T TK, Corydon L. Accuracy of the newer generation intraocular lens power calculation formulas in long and short eyes. J Cataract Refract Surg. 1991;17:187-93.

9. Savini G HK, Barboni P, Schiano Lomoriello D, Ducoli P. Corneal asphericity and IOL power calculation in eyes with aspherical IOLs. J Refract Surg. 2017;33:476-81.

10. Kane JX VHA, Atik A, Petsoglou C. Intra-ocular lens power formula accuracy: comparison of 7 formulas. J Cataract Refract Surg. 2016;42:1490-500.

11. Roessler GF DT, Plange $\mathrm{N}$ et al. Accuracy of intraocular lens power calculation using partial coherence interferometry in patients with high myopia. Ophthalmic Physiol Opt. 2012;32:228-33.

12. Pan CW RD, Saw SM. Worldwide preva-lence and risk factors for myopia. Ophthalmic Physiol Opt. 2012;32:3-16.

13. Melles RB HJ, Chang WJ. Accuracy of intraocular lens calculation formulas. Ophthalmology 2018;125:169-78.

14. Olsen $\mathrm{T}$ CL, Gimbel $\mathrm{H}$. Intraocular lens power calculation with an improved anterior chamber depth prediction algorithm. J Cataract Refract Surg. 1995;21:313-9.

15. GD. B. An improved universal theoretical formula for intraocular lens power prediction. J Cataract Refract Surg. 1993;19:713720. 
16. Formula. BUI. Singapore, Asia-Pacific Association of Cataract and Refractive Surgeons. [updated [Last cited on 2019 Feb 22].]. Available from: https://www.apacrs. org/barrett_universal2/.

17. Findl O DW, Menapace R, Heinzl H, Hitzenberger CK, Fercher AF.. Improved prediction of intraocular lens power using partial coherence interferometry. J Cataract Refract Surg. 2001;27:861-7.

18. Olsen T. The Olsen formula. In: Shammas HJ e, Intraocular Lens Power Calculations. Thorofare, NJ, Slack, 2004; 27-38.

19. Cooke DL CT. Comparison of 9 intraocular lens power calculation formulas. J Cataract Refract Surg. 2016;42:1157-64.

20. Masket S TD. Astigmatic stabilization of $3.0 \mathrm{~mm}$ temporal clear corneal cataract incisions. J Cataract Refract Surg. 1996;22:1451-5.

21. Barrett GD. Barrett Universal II Formula. Singapore A-PAoCaRSAahaobuAM, 2018.

22. PhacoOptics - IOL power calculation software. IOL Innovations ApS. Aarhus N DAahwpnAM, 2018.

23. Hoffer KJ AJ, Haigis W, Olsen T, Savini G, Shammas HJ, Bentow S.. Protocols for studies of intraocular lens formula accuracy [edito-rial]. Am J Ophthalmol 2015;160:403-5.

24. Wang Q JW, Lin T, Wu X, Lin H, Chen W. Meta-analysis of accuracy of intraocular lens power calculation formulas in short eyes. Clin ExperimentOphthalmol. 2017.

25. KJ. H. Clinical results using the Holladay 2 intra-ocular lens power formula. J Cataract Refract Surg. 2000;26:1233-7.

26. Holladay JT LM, Waring GO, et al. The relationship of visual acuity, refractive error, and pupil size after radial ker-atotomy. Arch Ophthalmol 1991;109:70-6.

27. Kane JX VHA, Atik A, Petsoglou C. Intraocular lens power formula accuracy: comparison of 7 formulas. J Cataract Refract Surg. 2016;42:1490-500.

28. Savini G HK, FACS, Balducci N, Barboni P, Schiano-Lomoriello D. Comparison of formula accuracy for intraocular lens power calculation based on measurements by a swept-source optical coherence tomography optical biometer. J Cataract Refract Surg 2020;46:27-33.

29. Gale RP SM, Johnston RL, Zuberbuhler B, McKibbin M. Benchmark standards for refractive outcomes after NHS cataract surgery.. Eye (Lond) 2009;23:149-52.

30. Melles RB KJ, Olsen T, Chang WJ.. Update on intraocular lens power calculation formulas. Ophthalmology 2019;126:1334-5.

31. Popovic M SM, Campos-Moller€ X, Pereira A, Ahmed IIK.. Wang-Koch formula for optimization of intraocular lens power calculation: Evalua-tion at a Canadian center. J Cataract Refract Surg. 2018;44:17-22.

32. Abulafia A BG, Rotenberg M, Kleinmann G, Levy A, Reitblat O, Koch DD, Wang L, Assia El.. Intraocular lens power calculation for eyes with an axial length greater than $26.0 \mathrm{~mm}$ : comparison of formulas and methods. J Cataract Refract Surg. 2015;41:548-56.

33. Hill DC SS, Hill CS, King TS, Scott IU, Ernst BB, Pantanelli SM.. In-traoperative aberrometry versus preoperative biometry for intraocular lens power selection in axial myopia. J Cataract Refract Surg. 
2017;43:505-10.

34. Pitault G LC, Leroux les Jardins S, Auclin F, Chong-Sit D, Baudouin C.. Biometrie optique des yeux avec implants phaques [Optical biometry of eyes corrected by phakic intraocular lenses]. J Fr Ophtalmol. 2005;28: 1052-7.

35. Sanders DR BD, Harton PJ Jr, Rivera RP. The Visian myopic Implantable Collamer Lens does not significantly affect axial length measurement with the IOLMaster. J Refract Surg. 2008;24:957-9.

36. Yu A ZL, Wang Q, Zhu S, Xue S, Su Y, Pan R.. [Effects of posterior chamber phakic intraocular lens on axial length measurements] [Chinese]. Zhonghua Yan Ke Za Zhi. 2015;51:206-9.

37. Shajari M KC, Petermann K, B€ohm M, Herzog M, de'Lorenzo N, Sch€onbrunn S, Kohnen T. Comparison of 9 modern intraocular lens power calculation formulas for a quadrifocal intraocular lens. J Cataract Refract Surg. 2018;44:942-8.

38. Wang L SM, Ma XJ, Kohnen T, Koch DD.. Optimizing intraocular lens power calculations in eyes with axial lengths above $25.0 \mathrm{~mm}$. Cataract Refract Surg. 2011;37:2018-27.

39. Amro M CW, Arej N, Jarade E. Third- and fourth-generation formulas for intraocular lens power calculation before and after phakic intraocular lens insertion in high myopia. J Cataract Refract Surg 2018;44(11):1321-5

40. Shin JY LJ, Seo KY, Kim EK, Kim T.. Comparison of preoperative and postoperative ocular biometry in eyes with phakic intraocular lens implanta-tions. Yonsei Med J. 2013;54:1259-65. 